\title{
ON THE APPLICABILITY OF THE WANG TRANSFORM FOR PRICING FINANCIAL RISKS
}

\author{
BY
}

\author{
Antoon Pelsser
}

\begin{abstract}
In an arbitrage-free economy, it is well-known that financial risks can be priced using equivalent martingale measures. We establish in this paper that, for general stochastic processes, the Wang Transform does not lead to a price which is consistent with the arbitrage-free price. Based on these results we must conclude that the Wang Transform cannot be a universal framework for pricing financial and insurance risks.
\end{abstract}

\section{INTRODUCTION}

In two recent papers, Wang (2000) and Wang (2002), Shaun S. Wang postulates a general framework for pricing financial and insurance risks. This framework is based on a distortion operator which acts on the probability distribution of the risk one seeks to price. This distortion operator is now known in the actuarial literature as the "Wang Transform".

In an arbitrage-free economy, it is well-known that financial risks can be priced using equivalent martingale measures. In this paper we investigate whether the Wang Transform is consistent with the arbitrage-free pricing approach for pricing financial risks. Wang shows that the Wang Transform is consistent with arbitrage-free pricing in the setting of Geometric Brownian Motion with constant coefficients. However, this result does not hold in general. We establish in this paper that, for general stochastic processes, the Wang Transform does not lead to a price which is consistent with the arbitrage-free price. Based on these results we must conclude that the Wang Transform cannot be a universal framework for pricing financial and insurance risks.

A related result has also been reported in Ruhm (2003). Using a different probabilistic setting, his paper proves the result that distribution-based pricing formulas for European-style payoffs cannot consistently produce arbitragefree prices. In this paper we consider the setting of continuous-time diffusion processes to derive our result.

JEL classification code: G13.

Astin Bulletin 38(1), 171-181. doi: 10.2143/AST.38.1.2030409 (C) 2008 by Astin Bulletin. All rights reserved. 
The remainder of this paper is organised as follows. In Section 2 we give a very brief introduction to stochastic processes, stochastic calculus and the related partial differential equations. In Section 3 we provide a brief introduction to arbitrage-free pricing. Armed with this knowledge we then proceed in Section 4 with our main result: the analysis of the Wang Transform. First we analyse which change of probability measure is actually implied by the Wang Transform. Then we analyse under which conditions the "Wang change of measure" is consistent with the change of measure required for arbitrage-free pricing. A simple example where the Wang Transform is not consistent with arbitragefree pricing is provided in Section 5.

\section{Stochastic Processes}

In this section we provide a very brief introduction in stochastic processes and stochastic calculus. We will only review those results needed for the proof of our claim in Section 4. All theorems in this section are provided without proofs. For a more formal introduction to this subject we refer to Karatzas \& Shreve (1991).

We begin with the premise that the evolution of traded assets' prices in an economy are described by stochastic differential equations. Suppose we have a traded asset $X_{t}$ in the economy whose price follows the stochastic differential equation

$$
d X_{t}=\mu\left(t, X_{t}\right) d t+\sigma\left(t, X_{t}\right) d W_{t}
$$

where $W_{t}$ denotes Brownian Motion. The $d W_{t}$-term can be interpreted as a "noise term" with the following properties: $\mathbb{E}\left[d W_{t}\right]=0$ and $\mathbb{E}\left[\left(d W_{t}\right)^{2}\right]=d t$. Please note that a realisation of a path $W_{t}$ of a Brownian Motion is a continuous function in time that is nowhere differentiable. As a consequence, the normal rules of integration and differentiation cannot applied to Brownian Motion.

As $X_{t}$ is the price of a traded asset, a financial derivative (or derivative) is an asset in the economy whose value at some time point $T>t$ is given by a function $f\left(X_{T}\right)$. Hence, the value $f\left(X_{T}\right)$ of the derivative at time $T$ is derived from the value of the underlying asset $X_{T}$. The expected value at time $t$ of the payoff $f\left(X_{T}\right)$ is given by the function $u(t, x)=\mathbb{E}\left[f\left(X_{T}\right) \mid X_{t}=x\right]$. The function $u(t, x)$ satisfies a partial differential equation:

Kolmogorov's Backward Equation. Let the stochastic differential equation for the process $X_{t}$ be given by (1). Define $u(t, x)=\mathbb{E}\left[f\left(X_{T}\right) \mid X_{t}=x\right]$. Then $u(t, x)$ is a solution to the partial differential equation

$$
\frac{\partial}{\partial t} u(t, x)+\mu(t, x) \frac{\partial}{\partial x} u(t, x)+\frac{1}{2} \sigma^{2}(t, x) \frac{\partial^{2}}{\partial x^{2}} u(t, x)=0
$$

for $t<T$ and boundary condition $u(T, x)=f(x)$. 
A special case of a derivative is the indicator variable $\mathbf{1}_{\left(X_{T} \leq y\right)}$. By taking an expectation of this indicator function we find the distribution function $F(t, x$; $T, y)$ of the process $X_{t}$ :

$$
F(t, x ; T, y)=\operatorname{Pr}\left[X_{T} \leq y \mid X_{t}=x\right]=\mathbb{E}\left[\mathbf{1}_{\left(X_{T} \leq y\right)} \mid X_{t}=x\right] .
$$

Hence, the distribution function also satisfies Kolmogorov's Backward Equation (2) with boundary condition $F(T, x ; T, y)=\mathbf{1}_{(x \leq y)}$.

Let the stochastic process $Y_{t}$ be defined as a function $Y_{t}=g\left(t, X_{t}\right)$ of the process $X_{t}$ for all $t$. Then the stochastic differential equation for $Y_{t}$ is given by:

Ito's Lemma. Let the stochastic differential equation for the process $X_{t}$ be given by (1). Let the function $g(t, x)$ be twice continuously differentiable. Define $Y_{t}=$ $g\left(t, X_{t}\right)$, then the stochastic differential equation for $Y_{t}$ is given by

$$
\begin{aligned}
d Y_{t}= & {\left[\frac{\partial}{\partial t} g\left(t, X_{t}\right)+\mu\left(t, X_{t}\right) \frac{\partial}{\partial x} g\left(t, X_{t}\right)+\frac{1}{2} \sigma^{2}\left(t, X_{t}\right) \frac{\partial^{2}}{\partial x^{2}} g\left(t, X_{t}\right)\right] d t } \\
& +\sigma\left(t, X_{t}\right) \frac{\partial}{\partial x} g\left(t, X_{t}\right) d W_{t} .
\end{aligned}
$$

Another concept we need to introduce is a martingale. A martingale is a stochastic process that stays on average at its current level. If we would track the gains and losses from a fair game over time, then we expect on average not to gain or loose money systematically. This is an example of a martingale. In mathematical terms we define:

Martingale. A stochastic process is a martingale if and only if

$$
\mathbb{E}\left[X_{T} \mid X_{t}=x\right]=x \quad \forall T \geq t .
$$

A stochastic differential equation with a $d t$-term equal to zero describes a martingale process ${ }^{1}$.

Because the Wang Transform is basically a method for changing the probability distribution of a stochastic process, we want to conclude this section with Girsanov's Theorem. This is a fundamental result in stochastic analysis. The Girsanov Theorem states that if we change the probability distribution of a stochastic process, we only change the $d t$-coefficient of the stochastic differential equation. Hence, our mathematical analysis of the Wang Transform will be built on the implied Girsanov transformation of the stochastic process.

1 This statement is not entirely correct as we are ignoring the difference between "local martingales" and "true martingales", which is a mathematical technicality. In the remainder of this paper we are implicitly assuming that all martingales we encounter are "true martingales". 
Girsanov's Theorem. For any stochastic process $K_{t}$, such that $\int_{0}^{t} K_{s}^{2} d s<\infty$ with probability one, consider the stochastic process $R_{t}$ given by

$$
R_{t}=\exp \left\{\int_{0}^{t} K_{s} d W_{s}-\frac{1}{2} \int_{0}^{t} K_{s}^{2} d s\right\},
$$

where $W_{t}$ is Brownian Motion under the probability measure $\mathbb{Q}$. Define the probability measure $\mathbb{Q}^{*}$ as $d \mathbb{Q}^{*}=R_{t} d \mathbb{Q}$. Under the probability measure $\mathbb{Q}^{*}$ the process

$$
W_{t}^{*}=W_{t}-\int_{0}^{t} K_{s} d s
$$

is also a Brownian Motion.

The stochastic process $R_{t}$ is called the Radon-Nikodym derivative and is often denoted by $R_{t}=\frac{d \mathbb{Q}^{*}}{d \mathbb{Q}}$. With the help of Ito's Lemma it is straightforward to show that the Radon-Nikodym derivative $R_{t}$ is a martingale under the probability measure $\mathbb{Q}$ with stochastic differential equation $d R_{t}=K_{t} R_{t} d W_{t}$ with a $d t$ term is equal to 0 . The stochastic process $K_{t}$ is often called the Girsanov kernel or Girsanov exponent.

To analyse the impact of a change of probability measure on a stochastic process $X_{t}$ we proceed as follows. A change in probability measure implies a Radon-Nikodym derivative $R_{t}$. If we apply Ito's Lemma to the stochastic process $R_{t}$ we obtain its stochastic differential equation and we can infer the Girsanov kernel $K_{t}$. The stochastic differential equation for $X_{t}$ under the new probability measure $\mathbb{Q}^{*}$ can now be obtained by substituting $d W_{t}=d W_{t}^{*}+K_{t} d t$ (which is equation (5) rewritten in differential form). Let us consider an example: suppose the stochastic differential equation for $X_{t}$ is given by (1) under the probability measure $\mathbb{Q}$. Under the new probability measure $\mathbb{Q}^{*}$, the stochastic differential equation for $X_{t}$ is equal to

$$
\begin{aligned}
d X_{t} & =\mu\left(t, X_{t}\right) d t+\sigma\left(t, X_{t}\right)\left(d W_{t}^{*}+K_{t} d t\right) \\
& =\left(\mu\left(t, X_{t}\right)+\sigma\left(t, X_{t}\right) K_{t}\right) d t+\sigma\left(t, X_{t}\right) d W_{t}^{*}
\end{aligned}
$$

and we see indeed that the change in probability measure only affects the $d t$ term of the process $X_{t}$.

We can now use the Kolmogorov Backward Equation (2) to find the partial differential equation that describes the probability distribution of $X_{t}$ under the new probability measure $\mathbb{Q}^{*}$. This is used in Section 4 to find which change of probability measure is actually implied by the Wang Transform.

\section{Arbitrage-FreE PRICING}

Again, in this section we provide a very brief introduction into arbitrage-free pricing. All theorems in this section are provided without proofs. For a more formal introduction in this subject we refer to Björk (1998). 
To calculate the prices of financial derivatives, we can use a very elegant mathematical theory which is based on the elements of stochastic analysis outlined in the previous section.

Suppose we have a traded asset $X_{t}$ in the economy whose price follows the stochastic differential equation (1). Suppose we also have an asset $N_{t}$, which is an asset with a strictly positive price. We can choose the asset $N_{t}$ as a numeraire, i.e. as a unit of measurement. In the remainder of the paper, we will express the prices of all traded assets in terms of the numeraire. So, $X_{t}$ is the price of the traded asset expressed in units of $N_{t}$. The euro-value is therefore equal to $X_{t} N_{t}$.

The first property we want to impose on our economy is a no-arbitrage property. An arbitrage opportunity is a trading strategy which costs no money at time $t$ and has a non-negative value at time $T>t$ and a positive probability of a strictly positive value. Such an arbitrage opportunity would be a "moneymachine" which is clearly something we would like to avoid in modeling the economy. An economy that admits no arbitrage opportunities is called arbitragefree.

The second property we want to impose on an economy is the completeness property. In the previous section we defined a financial derivative as a traded asset which has a value of $f\left(X_{T}\right)$ at time $T$. Given the price processes of the two traded assets $X_{t}$ and $N_{t}$, we can ask the question whether it is possible to find a trading strategy using only $X_{t}$ and $N_{t}$ that replicates the value of the derivative $f\left(X_{T}\right)$ at time $T$. The economy is called complete if for any derivative such a replicating trading strategy exists.

If a replicating trading strategy can be found for any derivative $f\left(X_{T}\right)$ then, in order to avoid arbitrage opportunities, the price at time $t<T$ for the derivative is given by the value of the replicating trading strategy at time $t$. This is known as arbitrage-free pricing.

The question whether an economy is arbitrage-free and complete can be determined with the help of the following theorem:

Unique Martingale Measure. An economy is arbitrage-free and complete if and only if there exists a unique probability measure such that the prices of all traded assets divided by a numeraire are martingales.

Let us investigate the economy with $X_{t}$ and $N_{t}$ we have defined above. To establish that our economy is arbitrage-free and complete we must find a unique probability measure such that the process $X_{t}$ is a martingale. Suppose the process $X_{t}$ follows (1). If we apply a Girsanov transformation to the process $X_{t}$ with Girsanov kernel $K_{t}$, then the process follows (6) under the new measure $\mathbb{Q}^{*}$.

There is only one possible choice for the Girsanov kernel that nullifies the $d t$-coefficient and turns the process $X_{t}$ into a martingale:

$$
K_{t}=-\frac{\mu\left(t, X_{t}\right)}{\sigma\left(t, X_{t}\right)}
$$


and this expression is known as the market price of risk. This establishes that our economy is arbitrage-free and complete if and only if $\sigma\left(t, X_{t}\right) \neq 0 \forall t$.

Now that we have established that our economy is arbitrage-free and complete, we also know that the prices divided by $N_{t}$ for all traded assets will be martingales under the probability measure $\mathbb{Q}^{*}$. In particular, the price of a financial derivative divided by the numeraire $f\left(X_{T}\right) / N_{T}$ is also martingale under $\mathbb{Q}^{*}$. Let us denote this price process by $f_{t} / N_{t}$ for $t<T$. From the martingale definition we obtain that the price $f_{t} / N_{t}$ at time $t<T$ is given by

$$
\frac{f_{t}}{N_{t}}=\mathbb{E}^{*}\left[\frac{f\left(X_{t}\right)}{N_{T}} \mid X_{t}\right]
$$

where $\mathbb{E}^{*}\left[. \mid X_{t}\right]$ denotes an expectation under the measure $\mathbb{Q}^{*}$ conditional on observing the price of the asset $X_{t}$. This expectation can be evaluated using the probability distribution $F^{*}(t, x ; T, y)$ for the process $X_{t}$ under the measure $\mathbb{Q}^{*}$.

\section{WANG TRANSFORM}

In two recent papers, Wang (2000) and Wang (2002), an alternative framework for pricing financial risks is postulated. This framework is based on a distortion operator which acts directly on the probability distribution of the asset one seeks to price.

For the economy we have defined in the previous section, the distortion operator is defined as follows. Given the probability distribution $F(t, x ; T, y)$ for the traded asset $X_{t}$, the Wang Transform constructs a new distribution $F^{W}(t, x ; T, y)$ via the distortion operator

$$
F^{W}(t, x ; T, y)=\Phi\left(\Phi^{-1}(F(t, x ; T, y))-\lambda(t, T)\right)
$$

where $\lambda(t, T)$ is a deterministic function. The function $\Phi($.$) denotes the stan-$ dard normal distribution function.

The function $\lambda(t, T)$ is determined by adjusting the distribution $F^{W}(t, x ; T, y)$ in such a way that the martingale condition $\mathbb{E}^{*}\left[X_{T} \mid X_{t}=x\right]=x$ is fitted for all $T$. Hence, $\lambda(t, T)$ is obtained by solving (numerically) the equation:

$$
\begin{aligned}
& \mathbb{E}^{W}\left[X_{T} \mid X_{t}=x\right]=\int_{-\infty}^{\infty} X_{t} d F^{W}\left(t, x ; T, X_{T}\right)= \\
& \int_{-\infty}^{\infty} X_{t} d \Phi\left(\Phi^{-1}\left(F\left(t, x ; T, X_{T}\right)\right)-\lambda(t, T)\right)=\mathbb{E}^{*}\left[X_{T} \mid X_{t}=x\right]=x \quad \forall T>t
\end{aligned}
$$

As $F^{W}($.$) is a monotonic function in \lambda(t, T)$ this equation will always have a unique solution for all $T>t$ with initial condition $\lambda(t, t)=0$.

Before we proceed, let us briefly introduce the line of reasoning we will use. First we analyse which change of probability measure (i.e. which Girsanov 
Transformation) is actually implied by the Wang Transform. Then we analyse under which conditions the "Wang change of measure" is consistent with the change of measure required for arbitrage-free pricing.

To investigate which Girsanov Transformation is implied by the Wang Transform we have to deduce which stochastic differential equation gives rise to the distribution $F^{W}($.$) . We know that a Girsanov Transformation only affects$ the $d t$-coefficient of the stochastic process. Hence, using (6), the Girsanov Transformation implied by the Wang Transform will lead to the following stochastic differential equation for the process $X_{t}$ :

$$
d X_{t}=\left(\mu\left(t, X_{t}\right)+\sigma\left(t, X_{t}\right) K_{t}^{W}\right) d t+\sigma\left(t, X_{t}\right) d W_{t}^{W}
$$

where $W_{t}^{W}$ denotes Brownian Motion under the "Wang probability measure" $\mathbb{Q}^{W}$ and $K_{t}^{W}$ denotes the Girsanov kernel associated with the Wang Transform.

We also know that the distribution function $F^{W}(t, x ; T, y)$ satisfies Kolmogorov's Backward Equation (2):

$$
\begin{array}{r}
\frac{\partial}{\partial t} F^{W}(t, x ; T, y)+\left(\mu(t, x)+\sigma(t, x) K_{t}^{W}\right) \frac{\partial}{\partial x} F^{W}(t, x ; T, y) \\
+\frac{1}{2} \sigma^{2}(t, x) \frac{\partial^{2}}{\partial x^{2}} F^{W}(t, x ; T, y)=0 .
\end{array}
$$

Hence, we obtain the following expression for $\mu(t, x)+\sigma(t, x) K_{t}^{W}$ :

$$
\left(\mu(t, x)+\sigma(t, x) K_{t}^{W}\right)=\frac{\frac{\partial}{\partial t} F^{W}(t, x ; T, y)+\frac{1}{2} \sigma^{2}(t, x) \frac{\partial^{2}}{\partial x^{2}} F^{W}(t, x ; T, y)}{-\frac{\partial}{\partial x} F^{W}(t, x ; T, y)} .
$$

Given the expression (9) for the Wang Transform, we obtain the following expressions for the partial derivatives (note that we will suppress the arguments of $F(t, x ; T, y)$ and $\lambda(t, T)$ hereafter to lighten the notation):

$$
\begin{aligned}
& \frac{\partial}{\partial t} F^{W}(t, x ; T, y)=\frac{\phi\left(\Phi^{-1}(F)-\lambda\right)}{\phi\left(\Phi^{-1}(F)\right)}\left(\frac{\partial}{\partial t} F-\phi\left(\Phi^{-1}(F)\right) \frac{\partial}{\partial t} \lambda\right) \\
& \frac{\partial}{\partial x} F^{W}(t, x ; T, y)=\frac{\phi\left(\Phi^{-1}(F)-\lambda\right)}{\phi\left(\Phi^{-1}(F)\right)} \frac{\partial}{\partial x} F \\
& \frac{\partial^{2}}{\partial x^{2}} F^{W}(t, x ; T, y)=\frac{\phi\left(\Phi^{-1}(F)-\lambda\right)}{\phi\left(\Phi^{-1}(F)\right)}\left(\frac{\lambda}{\phi\left(\Phi^{-1}(F)\right)}\left(\frac{\partial}{\partial x} F\right)^{2}+\frac{\partial^{2}}{\partial x^{2}} F\right) .
\end{aligned}
$$

Substituting these expressions into (13) and simplifying yields: 


$$
\sigma(t, x) K_{t}^{W}=\left(\left(\frac{\phi\left(\Phi^{-1}(F)\right)}{\frac{\partial}{\partial x} F}\right) \frac{\partial}{\partial t} \lambda-\frac{1}{2} \sigma^{2}(t, x)\left(\frac{\frac{\partial}{\partial x} F}{\phi\left(\Phi^{-1}(F)\right)}\right) \lambda\right) .
$$

If we want to establish under which conditions the Wang Transform is consistent with arbitrage-free pricing, we can substitute the market price of risk (7) for $K_{t}^{W}$ and we obtain $\sigma(t, x) K_{t}^{W}=-\mu(t, x)$ and we can rewrite (14) as a differential equation in $\lambda(t, T)$ :

$$
\frac{\partial}{\partial t} \lambda(t, T)=-\left(\mu(t, x) \frac{\frac{\partial}{\partial x} F}{\phi\left(\Phi^{-1}(F)\right)}\right)+\frac{1}{2}\left(\sigma(t, x) \frac{\frac{\partial}{\partial x} F}{\phi\left(\Phi^{-1}(F)\right)}\right)^{2} \lambda(t, T) .
$$

This differential equation can be solved by $\lambda(t, T)$ if and only if the coefficients in the equation are functions of time only. We summarise this result in the following corollary:

Validity of Wang Transform. The Wang Transform is consistent with arbitragefree pricing if and only if the following conditions on $\mu(t, x)$ and $\sigma(t, x)$ are satisfied

$$
\frac{\partial}{\partial x}\left(\mu(t, x) \frac{\frac{\partial}{\partial x} F(t, x ; T, y)}{\phi\left(\Phi^{-1}(F(t, x ; T, y))\right)}\right)=0
$$

and

$$
\frac{\partial}{\partial x}\left(\sigma(t, x) \frac{\frac{\partial}{\partial x} F(t, x ; T, y)}{\phi\left(\Phi^{-1}(F(t, x ; T, y))\right)}\right)=0 .
$$

Note that these conditions are very restrictive. For example, a necessary condition for the coefficients in (15) to be functions of time only, is that the ratio $\mu(t, x) / \sigma(t, x)$ is a function of time only.

We must therefore conclude that the Wang Transform is, in general, not consistent with arbitrage-free pricing and can therefore not constitute a universal framework for pricing financial and insurance risks.

\section{COUNTER-EXAMPLE FOR WANG TRANSFORM}

Suppose we have an economy where the stock price process $S_{t}$ follows the stochastic differential equation:

$$
d S_{t}=\left(\mu+a\left(\mu t-\ln S_{t}\right)\right) S_{t} d t+\sigma S_{t} d W_{t}
$$


where $\mu, a$ and $\sigma$ are constants. Such a specification is consistent with a model for stock prices where the return on stock prices is mean reverting towards the level $\mu$. The speed of mean reversion of the returns is governed by the parameter $a$. Note that for $a=0$ the model is equal to the standard Black-Scholes model. Furthermore, let the bond price $B_{t}$ be given by

$$
d B_{t}=r B_{t} d t
$$

where the rate of interest $r$ is a constant. The bond has a strictly positive price and can be used as a numeraire. Hence, if we set $X_{t}=S_{t} / B_{t}$ then we have

$$
d X_{t}=\left(\mu-r+a\left((\mu-r) t-\ln X_{t}\right)\right) X_{t} d t+\sigma X_{t} d W_{t} .
$$

Note that this specification for the $X_{t}$ process does not satisfy the necessary condition for validity of the Wang Transform.

The distribution function $F(t, x ; T, y)$ is a solution of Kolmogorov's Backward Equation (2). If we consider the logarithm of the process $X_{t}$ defined here, the distribution function of $\ln X_{t}$ can be expressed in closed form as

$$
\begin{aligned}
& F(t, x ; T, y)=\Phi\left(\frac{y-m(t, x ; T)}{\frac{\sigma^{2}}{2 a}\left(1-e^{-2 a(T-t)}\right)}\right) \\
& m(t, x ; T)=e^{-a(T-t)} x+\int_{t}^{T} e^{-a(T-s)}\left(\mu-r-\frac{1}{2} \sigma^{2}+a(\mu-r) s\right) d s
\end{aligned}
$$

and we see that $\ln X_{T}$ has a normal distribution with mean $m(t, x ; T)$ and variance equal to $\frac{\sigma^{2}}{2 a}\left(1-e^{-2 a(T-t)}\right)$. Note that for $T \rightarrow \infty$ the variance is bounded by $\sigma^{2} / 2 a$.

The economy we have defined here is arbitrage-free and complete if and only if there is a unique probability measure $\mathbb{Q}^{*}$ such that the process $X_{t}$ is a martingale. It is easy to see that the process $X_{t}$ is a martingale only for the probability measure $\mathbb{Q}^{*}$ defined by the Girsanov kernel

$$
K_{t}=-\frac{\mu-r+a\left((\mu-r) t-\ln X_{t}\right)}{\sigma} .
$$

Under the martingale measure $\mathbb{Q}^{*}$ the price process $X_{t}$ follows the stochastic differential equation

$$
d X_{t}=\sigma X_{t} d W_{t}^{*}
$$


Under the measure $\mathbb{Q}^{*}$ the process $\ln X_{t}$ therefore has a distribution function given by

$$
F^{*}(t, x ; T, y)=\Phi\left(\frac{y-x}{\sigma^{2}(T-t)}\right) .
$$

In particular we see that the variance of $\ln X_{T}$ under the arbitrage-free pricing measure $\mathbb{Q}^{*}$ is given by $\sigma^{2}(T-t)$ which is not bounded for $T \rightarrow \infty$.

If we apply the Wang Transform we have to solve $\lambda(t, T)$ from equation (10). For the distribution function(21) we find the following solution for $\lambda(t, T)$ :

$$
\lambda(t, T)=\frac{x-m(t, x ; T)}{\frac{\sigma^{2}}{2 a}\left(1-e^{-2 a(T-t)}\right)}
$$

and we obtain the following distribution function for the process $X_{t}$ under the Wang Transform

$$
F^{W}(t, x ; T, y)=\Phi\left(\frac{y-x}{\frac{\sigma^{2}}{2 a}\left(1-e^{-2 a(T-t)}\right)}\right) .
$$

Note that under the Wang Transform, the variance of the process $X_{t}$ is different than the correct variance under the arbitrage-free pricing measure which may lead to considerable mispricing of financial risks based on the Wang Transform. This concludes our counter-example.

\section{ACKNOWLEDGEMENTS}

The author would like to thank seminar participants at the University of Bonn, the ICMS Workshop on the Interface between Quantitative Finance and Insurance, David Ruhm and an anonymous referee for comments and helpful suggestions. All remaining errors are the responsibility of the author.

\section{REFERENCES}

BuöRk, T. (1998) Arbitrage Theory in Continuous Time, Oxford University Press, Oxford. Karatzas, I. and S.E. Shreve (1991) Brownian Motion and Stochastic Calculus, Second Edition, Springer Verlag, New York.

Ruhm, D. (2003) "Distribution-Based Pricing Formulas Are Not Arbitrage-Free", Casualty Actuarial Society Proceedings, XC, 97-129.

WANG, S. (2000) "A Class of Distortion Operators for Pricing Financial and Insurance Risks", Journal of Risk and Insurance, 67(1), 15-36. 
WANG, S. (2002) "A Universal Framework for Pricing Financial and Insurance Risk", ASTIN Bulletin, 32(2), 213-234.

\section{Antoon Pelsser \\ University of Amsterdam and NETSPAR \\ Dept. of Quantitative Economics}

Roetersstraat 11

1018 WB Amsterdam

The Netherlands

Tel.: (31)20-525 4210

Fax: (31)20-525 4349

E-Mail:a.a.j.pelsser@uva.nl

Home-page: http://www.ase.uva.nllpplapelsser 